\title{
The chemical composition of Amazonian plants(")
}

\section{A Catalogue, edited by Setor de Fitoquimica, INPA, Manaus, Amazonas}

FAMILY :

Lauraceae

OCCURRENCE : Manaus, Amazonas

TXuNk woop :

anibine ( 1 )

(6S)-dihydrokawain ( Ila)

(6S)-dihydromethysticin ( Ilb)

(6S)-tetrahydroiangonin ( Ilc )

( $6 \mathrm{~S}$ )-tetrahydro-11-methoxyiangonin ( Ild)
SPECIE :

Aniba gigantifolia O. C. Schmidt

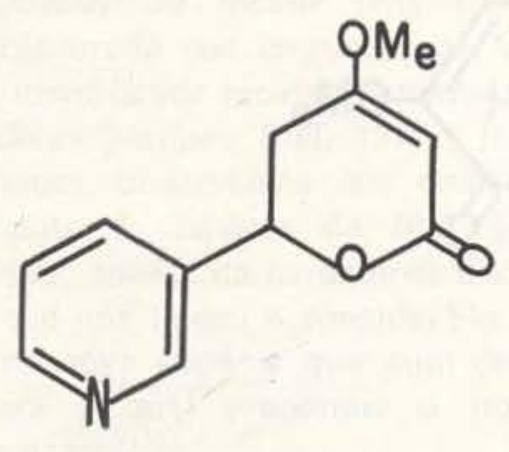

(1)

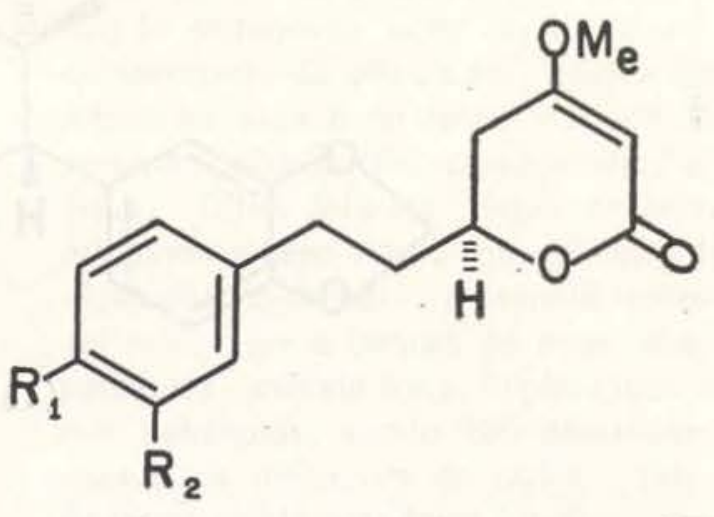

$\begin{array}{ll}\text { ( Ila) } & R_{1}=R_{2}=H \\ \text { (IIi) } & R_{1}=R_{2}=O \mathrm{OCH}_{2} \mathrm{O} \\ \text { (IIc) } & \mathrm{R}_{1}=\mathrm{OMe} ; \quad \mathrm{R}_{2}=\mathrm{H} \\ \text { (IId) } & \mathrm{R}_{1}=\mathrm{R}_{2}=\mathrm{OMe}\end{array}$

REFERENCES :

1. Von Bülow, M. V., Franca, N. C., Gottlieb, and Puentes Suarez, A. M. (1973) Phytochemistry 12, (no prelo)

2. Gottlieb, O. R. (1972) Phytochemistry 11, 1537

3. Saver, H. and Hänsel, R. (1967) Planta Med. 15, 443

4. Achenbach, H., Karl, W. and Smith, S. (1971) Chem. Ber. 104, 2688

(*) - Contributions to this catalogue, which will be continued in subsequent issues of this Journal, are invited, and should be submitted to adress given above. 
FAMILY :

Lauraceae
SPECIE :

Aniba guianensis Aubl.

OCCURRENCE : Manaus, Amazonas

TRUNK WOOD :

benzyl benzoate

benzyl salicylate

O-methyleugenol

O-methylisoeugenol

guianin (1-allyl-8-hidroxy-3-methoxy-7-methyl-4-oxo-6-piperonyl-bicyclo [ 3.2.1] oct-2-en) (1)

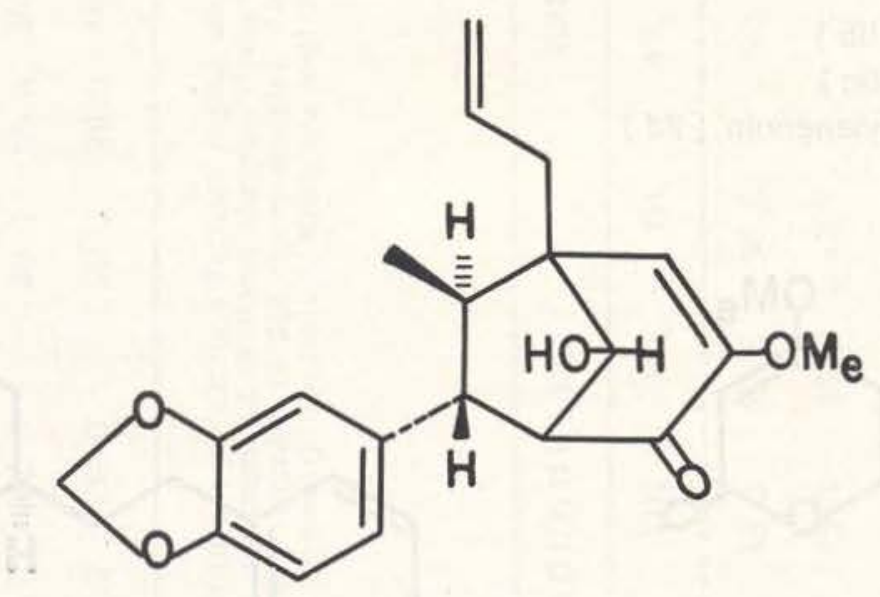

(1)

REFERENCES :

1. Andrade da Mata Rezende, C. M., Gottlieb, O. R., Leão da Silva, M. and Soares Maia, J. G. (1973) Phytochemistry 12, 471

2. Araujo Lima, O., Gottlieb, O. R. and Taveira Magalhães, M. (1972) Phytochemistry 11, 2031

3. Aiba, C. J., Braz Filho, R. and Gottlieb, O. R. (1973) Phytochemistry 12, 413

4. Gottlieb, O. R. (1972) Phytochemsitry 11, 1537. 